\title{
Epilepsy: Special Consideration in Women
}

\author{
${ }^{1}$ Ayesha Ahmad, ${ }^{2}$ Tamkin Khan Rabbani, ${ }^{3}$ Suman Kumar
}

\begin{abstract}
Epilepsy is one of the most common chronic neurological disorder complicating pregnancy. The article reviews various management issues in women in preconceptional, antenatal and postnatal period. An online search was carried out for articles on epilepsy in women and different aspects relating to contraception, conception, antenatal, intranatal and postnatal management. The available guidelines on the subject were also reviewed.
\end{abstract}

Keywords: Epilepsy, Anti-epileptic drugs, Seizures, Convulsion, WWE.

How to cite this article: Ahmad A, Rabbani TK, Kumar S. Epilepsy: Special Consideration in Women. Int J Infertil Fetal Med 2015; 6(1):11-14.

Source of support: Nil

Conflict of interest: None

Date of Received: 18-01-15

Date of Acceptance: 28-02-15

Date of Publication: April 2015

\section{INTRODUCTION}

Epilepsy is the most common neurological disorder affecting pregnant women and it is estimated to have a prevalence of $0.5 \%{ }^{1}$ At times, seizures may present for the first time during antenatal or postnatal period. Epilepsy, and drugs used for treatment have a significant impact on reproductive function of females. The disease and its management, therefore, becomes important for obstetricians and gynecologists as majority of women with epilepsy (WWE) require continuation of antiepileptic drugs (AEDs) prior to and during pregnancy.

\section{METHODOLOGY}

A review of the existing literature on issues in WWE was done including the latest available guidelines on the

\footnotetext{
${ }^{1}$ Assistant Professor, ${ }^{2}$ Professor, ${ }^{3}$ Consultant

${ }^{1}$ Department of Obstetrics and Gynecology, Hamdard Institute of Medical Science and Research, New Delhi, India

2Department of Obstetrics and Gynecology, JN Medical College Aligarh Muslim University, Aligarh, Uttar Pradesh, India

${ }^{3}$ Department of Obstetrics and Gynecology, Fortis Group of Hospitals, Gurgaon, Haryana, India

Corresponding Author: Ayesha Ahmad, Department of Obstetrics and Gynecology, JN Medical College, Aligarh Muslim University, Aligarh, Uttar Pradesh, India, e-mail: docayeshaahmad@gmail.com
}

subject. Based on the existing evidence, the authors have tried to formulate a management protocol for such women.

\section{SEIZURE RISK DURING PREGNANCY}

The incidence of seizures during pregnancy is around 0.15 to $10 \%$, with labor being an especially vulnerable period. The effect of pregnancy on seizure frequency is controversial. Around one third WWE will have an increase in frequency whereas around two thirds will experience either no change or even a decrease in frequency. ${ }^{4}$ Some of this can be attributed to physiologic adaptations and psychological stress associated with pregnancy. These include increased steroid hormone levels, sleep deprivation, decreased gastrointestinal absorption and increased hepatic and renal clearance of AEDs. Plasma albumin levels decrease in pregnancy, leading to lower total drug levels. At times, patient non compliance secondary to fear of effect of drugs on fetus may contribute to altered seizure frequency. ${ }^{5,6}$

The longer a woman has been seizure free, the lesser are the chances of her sustaining convulsions during pregnancy. Studies show that almost all women who have an average seizure frequency of more than one per month will worsen during pregnancy. This is in contrast to only $25 \%$ of women developing seizures if they have a seizure free interval of at least 9 months. ${ }^{7}$ In general, as long as compliance with treatment is assured, the seizure frequency is not increased in pregnancy. The risk of sustaining tonic clonic seizures, usually remains low during labor and 24 hours after birth.

The women need to be reassured that simple partial, complex partial, absence and myoclonic seizures have not been known to have deleterious effects on the pregnancy or the fetus, unless the patient falls and sustains an injury. However, generalized tonic clonic seizures may have adverse effects on the fetus, although the absolute risk is low and the level of risk may depend on seizure frequency.

\section{PHARMACOLOGICAL ASPECTS}

The use of AEDs is associated with an increased incidence of congenital anomalies in the fetus. The risk of congenital malformations in women on AEDs is around 6 to $8 \%$ (two to three times of general population). Multiagent therapy increases the risk of congenital malformations. Among women who take AEDs during pregnancy, the risk of 
congenital anomalies is higher in those who have already had a pregnancy with fetal malformation. ${ }^{8}$

The four most commonly used first line agents are carbamazepine, phenobarbital, phenytoin and valproic acid. All these drugs cross placenta and have teratogenic effects. All of them can cause fetal anticonvulsant syndrome, although some AEDs are particularly linked with certain anomalies. The fetal anticonvulsant syndrome consists of a cluster of anomalies including microcephaly, facial dysmorphism [including cleft lip and palate, hypertelorism, flat nasal bridge, low set abnormal ears, epicanthic folds, long philtrum], neural tube defects, congenital heart defects, intrauterine growth restriction and developmental delay in the infant. ${ }^{9,10}$ Some AEDs are enzyme inducers and may enhance their own metabolism and that of other agents (Table 1). ${ }^{11}$ Although valproate is a non enzyme inducing AED, it may inhibit the enzyme epoxide hydrolase, which metabolises phenytoin and carbamazepine.

The search for an ideal AED during pregnancy remains elusive. Newer drugs, such as lamotrigine, gabapentin and tiagabine have a good record in animal studies, but we have very limited experience in human studies. Carbamazepine is considered to be a safer AED. However, it has also been associated with a spina bifida rate of approximately $1 \%$. Sodium valproate is especially notorious for having risk of recurrent malformations. It is recommended that unless there is no effective alternative to it, sodium valproate should not be used during pregnancy and in women of childbearing potential. Valproate $^{12}$ exposure is associated with lower IQ levels, neurodevelopmental delay and poor verbal and memory abilities in children exposed to valproate in intrauterine life. Appropriate counseling should be provided to women who are being started on the drug or already taking it, weighing the benefits of treatment as against the risk of teratogenic and neurodevelopmental effects on children. In case valproate is the AED of choice, the total required drug can be given in divided doses to avoid high plasma concentrations. Alternatively, a slow release preparation can be administered.

Vitamin K deficiency is a poorly understood feature of AEDs (carbamazepine, phenytoin, phenobarbitone,

Table 1: Classification of antieplileptic drugs on the basis of enzyme inducing activity

\begin{tabular}{ll}
\hline Enzyme-inducing AEDs & Nonenzyme-inducing AEDs \\
\hline Phenobarbitone & Valproate \\
Phenytoin & Lamotrigine \\
Carbamazepine & Clonazepam \\
& Ethosuximide \\
& Gabapentin \\
\hline
\end{tabular}

AEDs: Antiepileptic drugs valproate). It may lead to hemorrhagic disease of the newborn, with catastrophic intracranial and gastrointestinal bleeding in some babies. This can be averted by maternal therapy with vitamin $\mathrm{K}$ supplements in the third trimester and administration of Vitamin $\mathrm{K}$ to the neonate. Phenytoin and carbamazepine have also been linked to an increased risk of childhood cancers, such as neuroblastoma.

It is recommended that when AEDs are initiated in women and girls of childbearing age, the risk of malformations and possible neurodevelopmental impairments in the fetus be discussed with the patients and/or their care providers. Accurate information should be imparted to WWE and their partners regarding conception, pregnancy, breastfeeding, children's health, contraception and menopause. All WWE need to informed that they are at a higher risk of complications during pregnancy and labor as compared to women who donot suffer from the disease. The possibility of status epilepticus and sudden unexpected death in pregnancy (SUDEP) needs to be informed especially in WWE who plan to discontinue AED therapy.

\section{PRECONCEPTUAL MANAGEMENT}

The best management of epilepsy in pregnancy begins with the counseling of prospective parents when they are planning conception. ${ }^{13}$ Ideally WWE need to be reviewed prior to planning a pregnancy and a plan of management charted in consultation with a neurologist. The medication needs to be reviewed in case a modification in the AEDs or reduction in the number of drugs is required. It is preferred to have a woman seizure free for at least 2 years before planning a pregnancy. In these women, supervized withdrawal of AEDs over a 3 to 6 months period may be deemed appropriate by the consultant neurologist. In other women, switching over to a monotherapy regimen instead of polytherapy may be attempted.

Folic acid, in a dose of $5 \mathrm{mg}$ per day needs to be administered for 3 months preconception and should be continued for at least 12 weeks after conception. Some doctors may consider administering folic acid throughout the pregnancy to avoid the risk of folate deficiency anemia.

\section{ANTENATAL MANAGEMENT}

All pregnancies in WWE should be registered as early as possible and have a multidisciplinary management. In case a woman presents when she is already pregnant, especially after first trimester, the neurologist may often continue the regimen she is taking as there is probably little benefit, the period of organogenesis being complete. In all cases, the basic aim of antenatal care is optimization of seizure control to a regimen where minimum number 
of drugs are given at the lowest dosage that affords protection against seizures. If the seizure frequency increases, compliance with treatment should be reviewed and a search for precipitating factors be carried out.

In case of poor compliance, the woman should be counseled that that non-adherence to AED may present a greater risk to the developing fetus than AED exposure. The importance of adequate sleep should also be emphasized to the women. If poor compliance has been excluded, it might be an indication for increasing the dosage of existing AEDs and/or addition of a new AED. Although routine monitoring of AED levels in pregnancy is not recommended, it might be attempted in these cases.

1. Screening for fetal malformations: A prenatal screening for congenital anomalies is recommended with nuchal translucency scan and serum screening. A detailed anomaly scan should be done at 18 to 20 weeks and fetal echocardiography at 22 weeks.

2. Preterm labor: In case the woman needs to be administered betamethasone, the dose of should be doubled to $24 \mathrm{mg}, 24$ hours apart as steroid metabolism is potentiated by enzyme inducers.

3. Administeration of Vitamin K in third trimester: In WWE taking enzyme inducing drugs, oral Vitamin K (20 mg per day) from 36 weeks onwards is recommended until delivery. In women at risk of preterm labor, earlier commencement of Vitamin $\mathrm{K}$ should be considered.

- Intranatal management: It is extremely important to document anticonvulsant drug history and inform the pediatrician when the woman is in labor.

- Decision for mode of delivery: Epilepsy per se is not a contraindication to vaginal delivery. An elective cesarean section may be considered in women refractory to treatment during the third trimester or in those who a prone to develop status epilepticus with significant stress. An emergency cesarean section may be decided in women who develop refractory status epilepticus during labor due to the risk of fetal asphyxia with prolonged or repeated seizures. At times repeated absence seizures may limit maternal awareness and ability to cooperate and cesarean delivery may be resorted to.

- Labour analgesia: Epidural analgesia is good for WWE. Extreme caution should be exercised in administering Pethidine as it may have a convulsant effect in some women.

- AEDs during labor: The woman's oral anti epileptic regimen should be continued during labor. In case this is not possible due to nausea or vomiting or after anesthesia, phenytoin can be given intravenously (Initial dose of $10 \mathrm{mg} / \mathrm{Kg}$, followed 2 hours later by a second dose of $5 \mathrm{mg} / \mathrm{Kg}){ }^{14}$
- Management of acute seizures: The drug of choice is Lorazepam, administered in $2 \mathrm{mg}$ boluses every 5 minutes as necessary. In case of nonavailability of the drug, diazepam may be used in bolus doses of 5 to $10 \mathrm{mg}$. In case of there being any doubt regarding cause of intrapartum seizure, whether due to Eclampsia or epilepsy, then, in addition to intravenous lorazepam or diazepam, a slow intravenous bolus of $4 \mathrm{gm}$ magnesium sulfate followed by $1 \mathrm{gm} /$ hour should be given. If seizures persist, the woman should be managed as for status epilepticus. The woman should be put on continuous cardiotocographic monitoring for at least 30 minutes following any seizure.

\section{POSTNATAL MANAGEMENT}

Anti epileptic drug levels should be monitored in the postnatal period because reversal of the physiologic changes of pregnancy leads to increased serum levels of AEDs. Usually, the dose should be decreased to prepregnancy levels after delivery. Mothers should be counseled about getting adequate sleep as sleep deprivation can precipitate seizures. Although the risk of injury to the neonate as a result of maternal seizures is low, appropriate advice should be given regarding suitable settings for feeding and changing baby nappies, minimal carrying of baby by the mother, using a pram with automatic brakes, etc. Advice regarding contraception and preconceptual care in subsequent pregnancy should also be given. A follow-up should be done with a consultant neurologist 3 to 4 months postdelivery.

Neonates of mothers taking AEDs are at increased risk of developing hemorrhagic disease of the newborn. Therefore, they should be administered Vitamin $\mathrm{K}, 1 \mathrm{mg}$ IM, after birth routinely.

- Breast feeding: All AEDs are secreted in breast milk. However, breastfeeding is usually not contraindicated for most agents as the resulting dose delivered to the infant is usually subtherapeutic. Phenobarbital is generally avoided because it causes greater neonatal sedation and neonatal withdrawal may occur on weaning, presenting with hyperexcitability, tremor, high-pitched cry, feeding problems and continual hunger. ${ }^{15}$ Diazepam is also to be used with caution as the effects on nursing infants are poorly elucidated. Lamotrigine is not a preferred drug in lactating mothers. In case the baby is unusually sleepy, the mother is advised to take AEDs after breast feeding.

\section{CONTRACEPTION}

Women with epilepsy should be given detailed advice regarding different contraceptive choices available to 
them, the risks and benefits of different contraceptive methods especially drug interactions with enzyme inducing drugs. Additional barrier methods need to be discussed in women taking enzyme inducing drugs and opting for hormonal contraception.

In women taking enzyme inducing AEDs, the minimum initial dosage of estrogen in the combined oral contraceptive pill (COC) should be $50 \mu \mathrm{g}$. In case of breakthrough bleeding, the dosage of estrogen may need to be increased to 75 or $100 \mu g$ per day. Tricycling is another option, wherein a woman takes three packs of $\mathrm{COC}$ without a break. Such a regimen provides enhanced contraceptive cover and can also reduce the frequency of seizures if they are hormonally triggered. ${ }^{16}$

Progesterone only pill (POP) and progesterone implants are not recommended as reliable contraceptives in women taking enzyme inducing AEDs. However, depot injections of progesterone can be administered, the repeat doses being given at shorter injection intervals (10 weeks instead of 12 weeks). Levonorgestrel-releasing intrauterine system (LNG-IUS) is recommended as the locally administered progestogen will not be affected by induced liver enzymes.

For the purpose of emergency contraception, an intrauterine contraceptive device is the most reliable choice in women on AEDs. In case a woman on enzyme inducing AEDs opts for hormonal methods, the recommended dose of levonorgestrel is increased to 1.5 and $750 \mu \mathrm{g}$ 12 hours apart.

\section{CONCLUSION}

There are multiple facets involved in management of epilepsy in women, right from preconceptual stage to pregnancy and childbirth, and further on to lactation and contraception. The management is multidisciplinary with involvement of obstetrician, neurologist and fetal medicine specialist, and a basic knowledge is mandatory for all the medical personnel involved in care of such women.

\section{REFERENCES}

1. Donaldson JO. Neurological disorders. In: de Swiet M, editor. Medical disorders in obstetric practice. 4th ed. Oxford, UK: Blackwell Publishing; 2002.
2. To WK, Cheung RTF. Neurological disorders in pregnancy. HKMJ 1997;3(4):400-408. Available at: http://hub.hku.hk/ bitstream/10722/53402/1/30242.pdf?accept $=1$

3. Adab N, Chadwick DW. Management of women with epilepsy during pregnancy. The obstetrician and gynaecologist. 2006;8:20-25. Available at: www.rcog.org.uk/togonline

4. Epilepsy guidelines group. Primary care guidelines for the management of females with epilepsy. London: Royal Society of Medicine Press Ltd; 2004.

5. Schmidt D. Pharmacotherapy of epilepsy-current problems and controversies. Fortschr Neurol Psychiatr 1983;51(11): 363-386.

6. Pennell PB. Antiepileptic drugs during pregnancy: what is known and which AEDs seem to be safest? Epilepsia 2008; 49(9):43-55.

7. Knight AH, Rhind EG. Epilepsy and pregnancy. A study of 153 pregnancies in 59 patients. Epilepsia 1975;16(1):99-110.

8. Holmes LB, Harvey EA, Coull BA, et al. The teratogenicity of anticonvulsant drugs. N Engl J Med 2001;344:1132-1138.

9. Moore SJ, Turnpenny P, Quinn A, et al. A clinical study of 57 children with fetal anticonvulsant syndromes. J Med Genet 2000;37:489-497.

10. Nelson-Piercy C. Handbook of obstetric medicine. 4th ed. Informa Healthcare; 2010. Chapter 9, Neurological Problems p. 156-162.

11. Kenny L. Neurological conditions. Obstetrics and gynaecology. In: Luesley DM, Baker PN, editors. An evidence based text for MRCOG. 2nd ed. London. Edward Arnold (Publish). 2010. Chap 6.10 p. 113-117.

12. Meador KJ, Baker GA, Browning N, Clayton-Smith J, CombsCantrell DT, Cohen M, et al. Cognitive Function at 3 years of age after fetal exposure to antiepileptic drugs. N Engl J Med 2009;360(16):1597-1605.

13. Delgado-Escueta AV, Janz D. Consensus guidelines: preconceptional counseling, management, and care of the pregnant woman with epilepsy. Neurol 1992;42(5):149-160.

14. Ryan G, Lange IR, Naugler MA. Clinical experience with phenytoin prophylaxis in severe preeclampsia. Am J Obstet Gynecol 1989;161(5):1297-1304.

15. Gaily E, Granstrom M-L. A transient retardation of early postnatal growth in drug-exposed children of epileptic mothers. Epilepsy Res 1989;4:147-155.

16. National Institute for Health Care and Excellence clinical guideline 137. The epilepsies: the diagnosis and management of the epilepsies in adults and children in primary and secondary care: NICE; 2012 [modified 2015]. Available at: http://www.nice.org.uk/guidance/cg137/resources/ guidance-the-epilepsies-the-diagnosis-and-managementof-the-epilepsies-in-adults-and-children-in-primary-andsecondary-care-pdf 